\title{
Statistical Linearization of Phased Arrays Using Power Adaptive Power Amplifier Model
}

\author{
Bilal Khan, Nuutti Tervo, Aarno Pärssinen and Markku Juntti \\ Faculty of Information Technology (ITEE), University of Oulu, Finland.
}

\begin{abstract}
Phased arrays used in millimeter-wave systems challenge the concept of power amplifier (PA) linearization by digital predistortion (DPD). This is due to the shared digital path and inaccuracies in analog beamforming and other component variations. However, the group behavior of multiple parallel nonlinear branches can be expected to be more predictable due to averaging effect compared to a single branch behavior. In this paper, we use a power adaptive nonlinear model to mimic the average behavior of a single $P A$ and utilize the probability distribution of the input power of each individual PA to approximate the expected nonlinear behavior of the array over-the-air. The approximated array response is used for the DPD training. The simulation results indicate that the proposed approach provides good linearization performance for large arrays that have varying amplitude and phase weights.
\end{abstract}

\section{INTRODUCTION}

The high data rate demand of the next generation communication systems require efficient utilization of higher frequency bands up to millimeter-wave (mmW) frequencies and even higher. The physical size of a single antenna element is small at such frequencies thus allowing to pack multiple antenna elements in a compact form factor. Multiple antennas are often implemented as phased arrays that are used to compensate for the high path loss of a mmW signal [1].

In order to deliver a decent transmission power for the antennas with a good power efficiency and low routing loss, practical array implementations have often an individual transmit power amplifier (PA) driving each antenna element. The power efficiency is desired not only for reducing the power consumption of the transmitter but also for reducing the thermal problems that are often severe due to the densely packed PAs used in mmW systems [2]. Hence, high-efficiency PAs are needed in $\mathrm{mmW}$ phased arrays to reduce the power consumption and to improve the reliability of the devices to increase their life-time. However, PA efficiency is usually at maximum when it is operating close to its peak power where the behavior of the PA is nonlinear. The nonlinearity of the PA results to in-band distortion (which degrades bit error rate) and out-of-band emissions (which causes interference to other users in adjacent frequency bands) [3].

Digital predistortion (DPD) can be used to compensate for the PA nonlinearity. However, analog beamforming arrays with multiple parallel PAs each having different phase and amplitude weights challenges the DPD concept. This is due to the fact that the parallel PAs have only one shared digital input and hence a single DPD has to be shared by multiple PAs. The nonlinear characteristics of the PAs are not identical due to several reasons. First, the PAs of the array can be different due to the implementation tolerances, temperature gradient and process variations. Second, in circulator-free array implementations [4], the PAs sees a load which varies over the steering angle due to the finite antenna coupling [5]. Even if the PAs are identical, their input powers may differ due to the implementation of the $\mathrm{mmW}$ power division network with phase shifters. The input powers over the branches may vary also with the steering angle due to the phase shifter control-word dependent gains [6], [7]. In addition, the input power per PAs can be purposely changed in order to shape the beam with variable gain amplifiers (VGAs) at each antenna branch e.g. in the case of amplitude tapering or zero forcing beamforming.

Several techniques for learning the nonlinear behavior of an array have been reported in the recent literature. In [8], DPD for fully digital beamforming system having PAs with different characteristics is presented. However, the input power per PA is assumed to be constant. In [9][11] the behavior of each individual PA is learned by switching the PAs to a single feedback path at different time instants. The learned PA or DPD models can be used to perform DPD over the PAs as in [11] or the array can be modelled in the far-field as in [9]. However, this process is time consuming and is not possible for real time operation especially if the number of PAs is large. In [7] and [12], the PA outputs are summed in analog domain to mimic the array behavior in the intended transmission direction. The used feedback receiver is called as phased feedback [7] or anti-beamforming network [12] and it enables continuous update of the DPD without the knowledge of the individual PA behaviors. However, the array feedback is complex to implement and the finite accuracy of the analog weighting is expected to have major impact on the DPD performance.

Due to the large number of antenna elements in $\mathrm{mmW}$ phased arrays, the array is able to average some of the variations out [7]. Hence, even if the parallel PA 
branches have varying nonlinear behavior, the group response of the PAs can be predicted by using the statistics of the variations over the parallel branches. This may even simplify the DPD procedure due to the fact that the DPD process can be made independent on the beamforming. In this paper, we (i) extend the poweradaptive model of [13] to mimic the average behavior of a single PA, (ii) utilize the probability distribution of the input powers to approximate the statistics of the nonlinear behavior of the PAs and (iii) combine the statistical models in order to mimic the statistical behavior of the array. In (iii), the concept of virtual array is used to create the array model. By using the presented approach the array DPD can be performed in statistical sense and hence it is not required to be updated continuously.

\section{Statistical DPD ARchitecture for Phased ARRAY}

\section{A. Overview of the Array DPD Setup}

The block diagram of a phased array with a common DPD is presented in Fig. 1. The array has $M_{A}$ parallel antennas and PAs. In the simulations, we use a memoryless look-up table (LUT) PA model extracted from amplitude to amplitude modulation (AMAM) and amplitude to phase modulation (AMPM) measurements of a $13 \mathrm{GHz}, 45-\mathrm{nm}, 4$-stack complementary metal oxide semiconductor (CMOS) silicon on insulator (SOI) PA [14]. The PA simulation model is presented in Fig. 2(a). The antennas are assumed to be identical $\lambda / 2$ spaced patch elements aligned as uniform linear array (ULA) in horizontal plane. Throughout the paper, we use 100 $\mathrm{MHz}$, 64-quadrature amplitude modulated (QAM) signal with root-raised-cosine pulse shaping ( 0.35 roll-off).

For the PA behavior training, we use a power adaptive model presented in Section II-B. The model is trained offline by switching at least one of the PA outputs to the feedback at a time. Throughout this paper we assume that the PAs are identical, but their inputs have randomly varying root mean square (RMS) power depending on the beamforming angle. The virtual array concept is used to create the statistical array model and is shown in Section II-C, and the DPD coefficient calculation is revised in Section II-D.

\section{B. Power Adaptive PA Modelling}

The power differences over the parallel branches change the input power of the PAs. Hence, the nonlinear behavior of each parallel branch is different. The PA modelling is often done by using polynomial models which are valid only close to the corresponding operational point to which it is trained. Hence, a power adaptive model for the PA is required which is valid at different input power levels. In [13] a power adaptive model for DPD is used due to the rapid variation of PA input power in systems where adaptive transmitter power

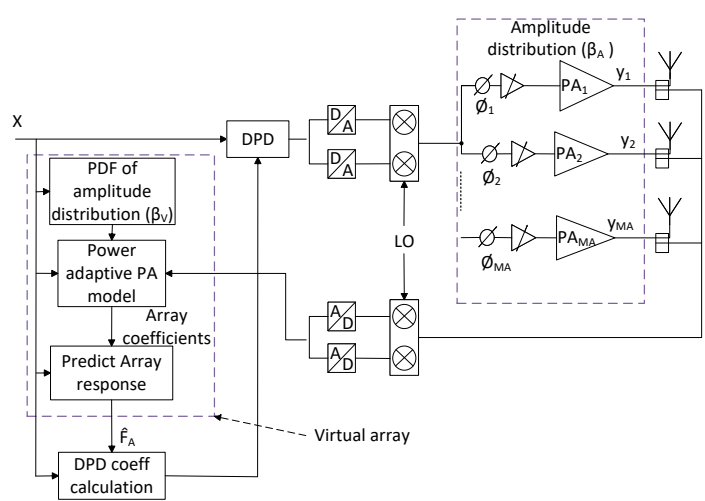

Fig. 1. Block diagram of the statistical array DPD system using virtual array and power adaptive PA model.

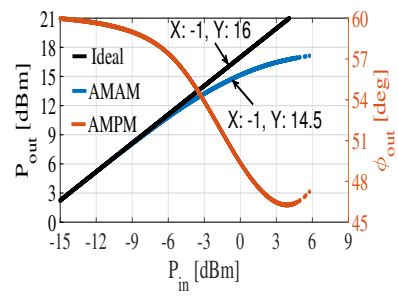

(a)

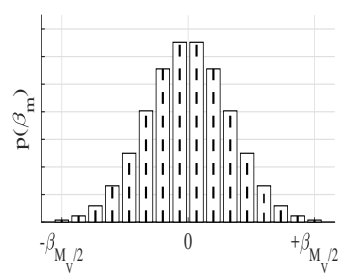

(b)
Fig. 2. (a) AMAM and AMPM responses of the PA simulation model and (b) example distribution with 16 virtual elements.

control is used. Here we extract the power adaptive PA model for large input power variation over the PA. Note that equation (4) is different from the mentioned reference due to the fact that we use the power adaptive model to model the PA and not the DPD.

The power adaptive model is created as follows. First, the PA input power range is divided into $L$ points, $P_{l}$, $l \in\{1,2, \ldots, L\}$, as shown in Fig 3 . The powers are arranged in a descending order as $P_{1}>P_{2}>\ldots>P_{L}$. In [13], the model is used to directly update the DPD coefficients based on the instantaneous RMS power. We use the same model to learn the nonlinear behavior of the PAs of the array. The PA coefficients $C^{(l)}$ with given input power $P_{l}$ can be updated as a function of power backoff from the $P_{1}$ as

$$
C^{(l)}=\hat{C}+\Delta C^{(l)},
$$

where

$$
\begin{gathered}
\Delta C^{(l)}=\left\{\begin{array}{l}
\alpha_{\operatorname{lin}}^{(l)} \Delta C_{\text {lin }} \\
\alpha_{\mathrm{n}-\operatorname{lin}}^{(l)} \Delta C_{\mathrm{n}-\mathrm{lin}}
\end{array}\right. \\
\alpha_{\operatorname{lin}}^{(l)}=G_{l}-G_{1}, \alpha_{\mathrm{n}-\operatorname{lin}}^{(l)}=\gamma_{l}-\gamma_{1},
\end{gathered}
$$

and

$$
\gamma_{l}=\sqrt{\frac{\sum_{n=1}^{N}\left|y(n)^{(l)}-G_{l} x(n)^{(l)}\right|^{2}}{\sum_{n=1}^{N}\left|y(n)^{(l)}\right|^{2}}} .
$$




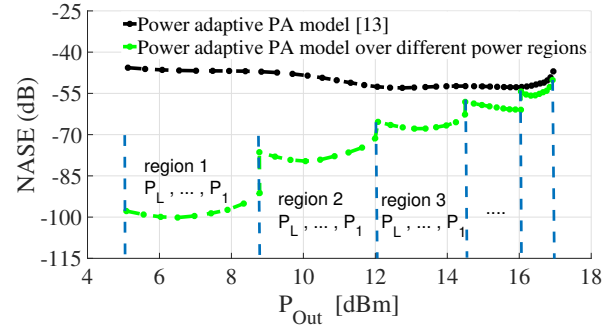

Fig. 3. Normalized average square error in power adaptive PA modelling.

In (1), $\hat{C}$ denotes the coefficient vector of the PA at reference power level $P_{1}$ and $\Delta C^{(l)}$ is the change in the PA coefficients at power level $P_{l}$ with respect to the reference power level $P_{1}$. In (2), $\Delta C_{\text {lin }}$ and $\Delta C_{\mathrm{n}-\mathrm{lin}}$ represent the linear and nonlinear coefficients of the PA, $\alpha_{\text {lin }}$ and $\alpha_{\mathrm{n}-\mathrm{lin}}$ are the scaling factors for linear and nonlinear part of the coefficient vector, respectively. In (3), $G_{l}$ is the gain of the PA at $P_{l}$ and $G_{1}$ is the gain of the PA at power level $P_{1}$. In (4), $\gamma_{l}$ is the measure of nonlinearity (MoN) of the PA at $P_{l}, \gamma_{1}$ is the MoN of the PA at $P_{1}, y(n)$ and $x(n)$ are the output and input signal of the PA with sampling index $n$ and $N$ is the number of time domain samples.

The performance of the power adaptive model of [13] can be improved for large input power range, by dividing the input power range into regions, with logarithmic spacing of $4 \mathrm{~dB}$ and use an individual power adaptive model of (1) - (4) at each of these regions. To demonstrate the performance of the extended power adaptive model, MATLAB simulation with the models presented in Section II-A is performed. The model is trained over 1024 samples with the modulated signal. The normalized average square error (NASE) of the output calculated as the difference between power adaptive model with respect to the PA LUT simulation model and is shown in Fig. 3.

\section{Statistical PA Array Model Training}

The proposed array DPD approach was shown in Fig. 1 . The amplitude distribution $\left(\beta_{A}=\left\{\beta_{1}, \beta_{2}, \ldots, \beta_{\mathrm{M}_{\mathrm{A}}}\right\}\right)$, causing the change in the input power of the PAs is assumed to be a set of independent and identically distributed Gaussian random variables in logarithmic scale with mean 0 and standard deviation $\sigma_{A}$. The virtual array under the dashed box is used to predict the array behavior and consist of three blocks. In the first block, the probability distribution function (PDF) of the amplitude distribution $\left(\beta_{V}\right)$ is sampled $M_{V}$ times as shown in Fig 2(b). We assume that the number of distribution samples equals to the number of array elements, i.e. $M_{V}$ $=M_{A}$. In the second block, the power adaptive PA model given in Section II-B is used to provide PA coefficients at each sample of $\left(\beta_{V}\right)$ in addition to the RMS power of

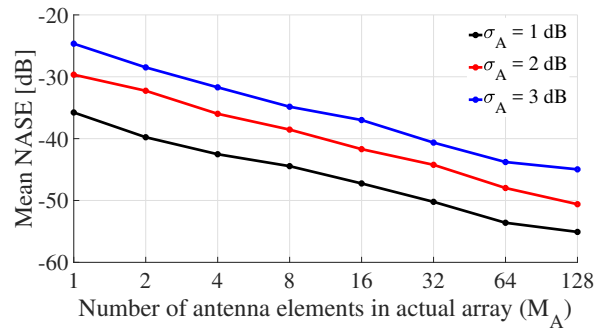

Fig. 4. Mean value of normalized average square error in array response prediction.

the common input. The PA coefficients are then scaled according to scaling factor

$$
S\left(\beta_{m}\right)=\frac{p\left(\beta_{m}\right)}{\sum_{m=1}^{M_{V}} p\left(\beta_{m}\right)} M_{V},
$$

where $p\left(\beta_{m}\right)$ is the probability of input amplitude $\beta_{m}$ in the virtual array. Hence, the expected value of the $k$ th order array coefficient can be written as

$$
C_{k}=\sum_{m=1}^{M_{V}} C_{m, k}^{(l)} S\left(\beta_{m}\right),
$$

where $C_{m, k}$ denotes the $m$ th sample of $k$ th coefficient. $l$ denotes the RMS power of input signal + sample of $\beta_{V}$. Finally, the predicted array far-field response is given by

$$
\hat{F}_{A}(n)=\frac{1}{G_{A}} \sum_{k=1}^{K} C_{k} x(n)|x(n)|^{k-1},
$$

where $G_{A}$ is the gain of the array and $K$ is the order of the polynomial used in the power adaptive model.

The performance of the virtual array model is simulated with different numbers of antenna elements in ULA. The simulations are performed over 500 Monte Carlo (MC) rounds by using the same PA model and waveform parameters as those in Section II-A. Fig. 4 shows the mean value of NASE in predicting the average array response with the presented virtual array approach. The simulation is run with different standard deviation of the input amplitude. The result shows that the modelling accuracy decreases as the standard deviation of the input amplitudes increase. However, the modelling error decreases linearly $3 \mathrm{~dB}$ every time when the number of antennas is doubled. Hence, more parallel PA branches we have, the better the statistical model performs.

\section{DPD Coefficient Extraction}

The expected array model derived in the previous sections is finally used to train the DPD. The predistorter model is an envelope power series with four coefficients. By using the predicted array response of (7), the predistorter input signal $x(n)$ can be written as

$$
x(n)=\sum_{k=1}^{K} d_{k} \hat{F}_{A}(n)\left|\hat{F}_{A}(n)\right|^{2(k-1)},
$$


where $\mathbf{d}=\left[d_{1}, d_{2}, \ldots, d_{K}\right], \mathbf{d} \in \mathbb{C}^{K}$ denotes the DPD coefficient vector, $K$ is the number of DPD coefficients, and $\hat{F}_{A}(n)$ is the array response predicted by (7). The problem can be written into matrix form and solved by least squares (LS) estimation as

$$
\mathbf{d}=\left(\mathbf{F}_{A}^{H} \mathbf{F}_{A}\right)^{-1} \mathbf{F}_{A}^{H} \mathbf{x},
$$

where $\mathbf{F}_{A} \in \mathbb{C}^{N \times \mathrm{K}}$, and include all the product terms of $F_{A}(n)\left|F_{A}(n)\right|^{2(K-1)}$ of (8), $\mathbf{x} \in \mathbb{C}^{N}$ is the common input signal, $N$ is the number of time domain samples and $(.)^{H}$ denotes the hermitian transpose.

\section{Statistical DPD Performance Analysis}

\section{A. Simulation Setup}

The performance of the proposed DPD approach is simulated for different sizes of ULAs. The used simulation model was presented in Section II-A and the DPD model is presented in Sections II-B-II-D. The average input power per PA is chosen to be $-1 \mathrm{dBm}$. This corresponds to PA output power of $14.5 \mathrm{dBm}$ as shown in Fig. 2(a). The RMS input amplitudes of the PAs are assumed to follow a Gaussian distribution in logarithmic scale with mean 0 and standard deviation $\sigma_{A}$. The simulations are performed for $\sigma_{A}=\{0,1,2,3\} \mathrm{dB}$ and the number of antenna elements in the array is varied as $M_{A}=\{1,2,4,8,16,32,64,128\} .500 \mathrm{MC}$ rounds with random steering angle $\theta_{s} \in \mathrm{U}\left(-40^{\circ}, 40^{\circ}\right)$, where $\mathrm{U}$ denotes the Uniform distribution, are simulated for each scenario. The used performance metrics are the adjacent channel power ratios (ACPR), the total radiated adjacent channel power ratio (TRACPR) and the error vector magnitude (EVM). The $\mathrm{ACPR}_{\max }$ and $\mathrm{TRACPR}_{\max }$ are presented as maximum of lower and upper adjacent channel powers. The ACPR ${ }_{\max }$ and EVM are measured at the steering angle while the TRACPR $R_{\max }$ is integrated over the space as proposed in [7].

As a reference scenario for the proposed DPD approach we use ideal phased feedback or antibeamforming network presented in [7], [12] which is used to continuously measure the array response and update the DPD coefficients for every MC run. Hence, in the reference scenario, the array response is assumed to be known at each time instant.

\section{B. Simulation Results}

Fig. 5, shows the mean value of the EVM for standard deviation of input amplitude $\sigma_{A}=\{0,1,3\} \mathrm{dB}$ for different numbers of antenna elements in the array. The phased feedback DPD approach is presented by red (solid and dashed) lines in the figure and is the reference DPD against the proposed virtual array-DPD. From the figure it can be seen that up to $1 \mathrm{~dB}$ of standard deviation of input amplitude variations, the virtual array-DPD performs the same as that of the phased feedback DPD. When the standard deviation

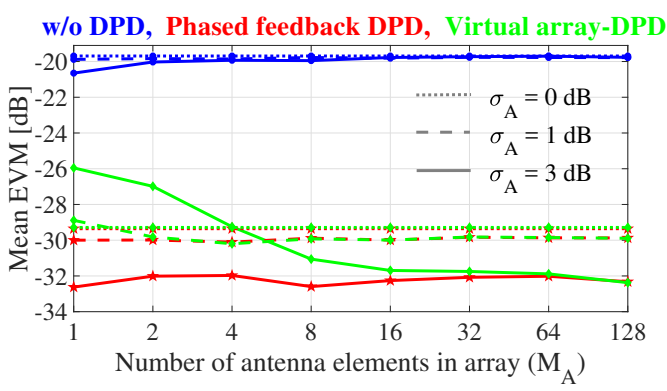

Fig. 5. Mean of EVM with different numbers of antenna elements and standard deviation values of PA input powers.

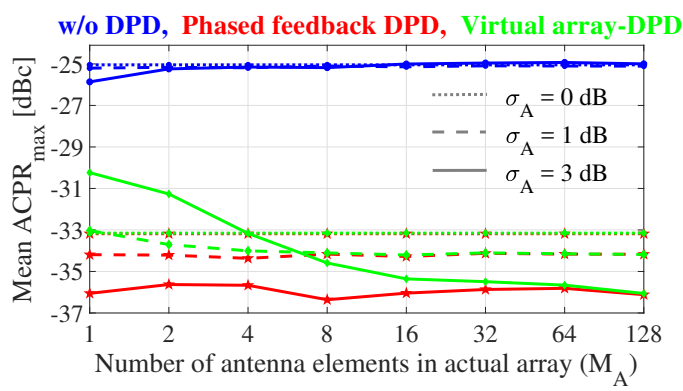

Fig. 6. Mean of $A C P R_{\max }$ with different numbers of antenna elements and standard deviation values of PA input powers.

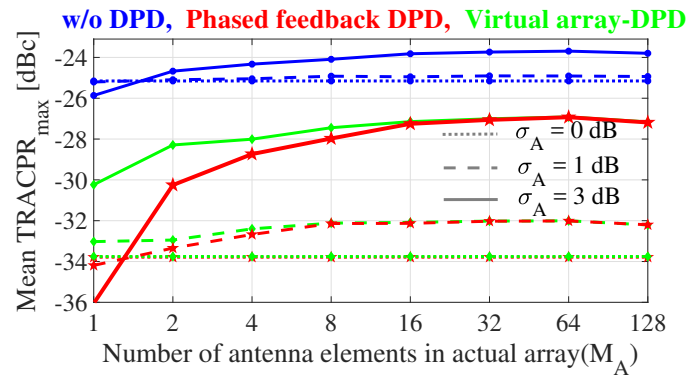

Fig. 7. Mean of TRACPR max $_{\text {ax }}$ with different numbers of antenna elements and standard deviation values of PA input powers.

of the amplitude variation increases up to $3 \mathrm{~dB}$, the performance of the virtual array-DPD deteriorates for small numbers of antenna elements in the array $\left(M_{A}\right)$. This is due to the fact that in small arrays, the array response is more random due to less averaging effect and thus the array nonlinearity strongly depend on the individual branch input amplitudes. However, in large antenna arrays, e.g. $M_{A} \geq 16$, the array behaviour is more predictable because the high number of elements averages the model towards the expected one due to the law of large numbers. Hence, the sampling mean converges to the expected mean and the virtual arrayDPD predicts the average array response with a good accuracy. Fig. 6 presents the $\mathrm{ACPR}_{\max }$ measured at the steering angle. The directed $\mathrm{ACPR}_{\max }$ behaves similarly as EVM.

From Figs. 5 and 6 it can be seen that in the 
presence of large amplitude differences the performance of both DPD (phased feedback and virtual array-DPD) is improved in terms of $\mathrm{ACPR}_{\max }$ and EVM. This is due to the fact that some of the PAs are driven hard into compression while other PAs are relatively linear [9]. If the array is modelled or measured correctly, the hard driven PAs are compensated for by the low driven PAs over-the-air. Hence, the in-band distortion creates a notch in the beamforming direction, thus improving $\mathrm{ACPR}_{\max }$ and EVM in the beamforming direction as proposed in [7]. However, the out-of-band distortion power is increased in other directions, thus affecting to the TRACPR $\mathrm{max}_{\text {max }}$ integrated over the space. Hence, the $\mathrm{TRACPR}_{\max }$ increases in the presence of larger variations as seen in Fig. 7.

The simulation results indicate that larger arrays can benefit from the differences over the nonlinearities even if the instantaneous RMS input powers are not known and the DPD is not trained for every beamforming angle. Moreover, the DPD process can be made independent on the beamforming as the larger arrays have less variance in the nonlinear behavior. In principle, this means that even relatively simple DPD schemes where a power adaptive model is trained offline or even extracted from simulations or factory measurements can achieve decent DPD performance that satisfies the specifications. As a reference, the current total radiated ACP specification in $3 \mathrm{GPP} / \mathrm{NR}$ standard is $28 \mathrm{dBc}$ for $28 \mathrm{GHz}$ frequency band. The ultimate solution would hence be that the feedback is not necessary or is required only for updating the power adaptive base model.

\section{CONCLUSION}

We utilize the statistical behavior of the PA array for training the DPD. The idea was to make DPD independent on beamforming such that it is not required to be updated continuously. We use a power adaptive nonlinear model to mimic the average behavior of a single PA, and utilize the probability distribution of the input powers to approximate the expected nonlinear behavior of the array over-the-air. The simulation results indicate that the statistical model can predict the array behavior with a good accuracy and it can be used to train the DPD for large arrays. On the contrary, smaller arrays require continuous feedback due to high variation in the nonlinear behavior of individual array branch. Similar concepts can be used for different beamformer distributions, component variations etc. The presented approach can be implemented as a slow process and the actual DPD of large arrays (16 elements or more), do not necessarily require constant update to fulfill the quite relaxed 3GPP NR ACPR requirements. In principle, this means that even a relatively simple DPD scheme, where a power adaptive model is trained offline, may produce decent results. The model can be even extracted from simulations or factory measurements.

\section{ACKNOWLEDGMENT}

This research work has been financially supported by Wireless Connectivity for Internet of Everything Energy Efficient Transceiver and System Design (WiConIE) and Academy of Finland 6Genesis Flagship (grant no. 318927).

\section{REFERENCES}

[1] K. Kibaroglu, M. Sayginer, and G. M. Rebeiz, "An Ultra Lowcost 32-element $28 \mathrm{GHz}$ Phased-array Transceiver with $41 \mathrm{dBm}$ EIRP and 1.01.6 Gbps 16-QAM Link at 300 meters," in 2017 IEEE Radio Frequency Integrated Circuits Symposium (RFIC), June 2017, pp. 73-76.

[2] C. Fager, K. Hausmair, T. Eriksson, and K. Buisman, "Analysis of Thermal Effects in Active Antenna Array Transmitters Using a Combined EM/Circuit/Thermal Simulation Technique," in 2015 Integrated Nonlinear Microwave and Millimetre-wave Circuits Workshop (INMMiC), Oct 2015, pp. 1-3.

[3] L. Guan and A. Zhu, "Green Communications: Digital Predistortion for Wideband RF Power Amplifiers," IEEE Microwave Magazine, vol. 15, no. 7, pp. 84-99, Nov 2014.

[4] A. Prata, S. C. Pires, M. Acar, A. S. R. Oliveira, and N. B. Carvalho, "Towards Circulator-free Multi Antenna Transmitters for 5G," in 2017 IEEE MTT-S International Microwave Symposium (IMS), June 2017, pp. 677-680.

[5] K. Hausmair, S. Gustafsson, C. Snchez-Prez, P. N. Landin, U. Gustavsson, T. Eriksson, and C. Fager, "Prediction of Nonlinear Distortion in Wideband Active Antenna Arrays," IEEE Transactions on Microwave Theory and Techniques, vol. 65, no. 11, pp. 4550-4563, Nov 2017.

[6] R. Mailloux, Phased Array Antenna Handbook, ser. Antennas and Propagation Library. Artech House, 2005.

[7] N. Tervo, M. E. Leinonen, J. Aikio, T. Rahkonen, and A. Pärssinen, "Analyzing the Effects of PA Variations on the Performance of Phased Array Digital Predistortion," in 2018 IEEE 29th Annual International Symposium on Personal, Indoor and Mobile Radio Communications (PIMRC), Sep. 2018, pp. 215-219.

[8] A. Brihuega and L. Anttila and M. Abdelaziz and M. Valkama, "Digital Predistortion in Large-Array Digital Beamforming Transmitters," in 2018 52nd Asilomar Conference on Signals, Systems, and Computers, Oct 2018, pp. 611-618.

[9] N. Tervo, J. Aikio, T. Tuovinen, T. Rahkonen, and A. Pärssinen, "Digital Predistortion of Amplitude Varying Phased Array Utilising Over-the-air Combining," in 2017 IEEE MTT-S International Microwave Symposium (IMS), June 2017, pp. 1165-1168.

[10] X. Liu, Q. Zhang, W. Chen, H. Feng, L. Chen, F. M. Ghannouchi, and Z. Feng, "Beam-Oriented Digital Predistortion for 5G Massive MIMO Hybrid Beamforming Transmitters," IEEE Transactions on Microwave Theory and Techniques, vol. 66, no. 7, pp. 3419-3432, July 2018.

[11] S. Lee, M. Kim, Y. Sirl, E. Jeong, S. Hong, S. Kim, and Y. H. Lee, "Digital Predistortion for Power Amplifiers in Hybrid MIMO Systems with Antenna Subarrays," in 2015 IEEE 81st Vehicular Technology Conference (VTC Spring), May 2015, pp. 1-5.

[12] M. Abdelaziz, L. Anttila, A. Brihuega, F. Tufvesson, and M. Valkama, "Digital Predistortion for Hybrid MIMO Transmitters," IEEE Journal of Selected Topics in Signal Processing, vol. 12, no. 3, pp. 445-454, June 2018.

[13] Y. Guo, C. Yu, and A. Zhu, "Power Adaptive Digital Predistortion for Wideband RF Power Amplifiers With Dynamic Power Transmission," IEEE Transactions on Microwave Theory and Techniques, vol. 63, no. 11, pp. 3595-3607, Nov 2015.

[14] J. P. Aikio, A. Sethi, R. A. Shaheen, R. Akbar, T. Rahkonen, and A. Pärssinen, "A Fully Integrated $13 \mathrm{GHz}$ CMOS SOI Stacked Power Amplifier for 5G Wireless Systems," in 2017 IEEE Nordic Circuits and Systems Conference (NORCAS): NORCHIP and International Symposium of System-on-Chip (SoC), Oct 2017, pp. $1-4$. 